\title{
BMJ Open Effects of cosmetic and other camouflage interventions on appearance-related and psychological outcomes among adults with visible differences in appearance: a systematic review
}

To cite: Gholizadeh S, Rice DB, Carboni-Jiménez A, et al. Effects of cosmetic and other camouflage interventions on appearance-related and psychological outcomes among adults with visible differences in appearance: a systematic review. BMJ Open 2021;11:e046634. doi:10.1136/ bmjopen-2020-046634

- Prepublication history and supplemental material for this paper is available online. To view these files, please visit the journal online (http://dx.doi. org/10.1136/bmjopen-2020046634).

Received 05 November 2020 Revised 15 February 2021 Accepted 17 February 2021

Check for updates

(c) Author(s) (or their employer(s)) 2021. Re-use permitted under CC BY-NC. No commercial re-use. See rights and permissions. Published by BMJ.

For numbered affiliations see end of article.

Correspondence to

Dr Brett D Thombs;

brett.thombs@mcgill.ca

\section{ABSTRACT}

Objective Visible differences in appearance are associated with poor social and psychological outcomes. Effectiveness of non-surgical cosmetic and other camouflage interventions is poorly understood. The objective was to evaluate effects of cosmetic and other camouflage interventions on appearance-related outcomes, general psychological outcomes and adverse effects for adults with visible appearance differences. Design Systematic review.

Data sources MEDLINE (Ovid), EMBASE (Ovid), PsycINF0 (Ovid) CINAHL and Cochrane Central databases searched from inception to 24 October 2020. Two reviewers independently reviewed titles and abstracts and full texts. Eligibility criteria Randomised controlled trials in any language on non-surgical cosmetic or other camouflage interventions that reported appearance-related outcomes, general psychological outcomes or adverse effects for adults with visible appearance differences.

Data extraction and synthesis Two reviewers independently extracted data, assessed intervention reporting using the Template for Intervention Description and Replication checklist, and assessed risk of bias using the Cochrane risk of bias tool. Outcomes included appearance-related outcomes, general psychological outcomes (eg, depression, anxiety) and adverse effects. Results One head-to-head trial and five trials with waiting list or routine care comparators were included. All had unclear or high risk of bias in at least five of seven domains. Effect sizes could not be determined for most outcomes due to poor reporting. Between-group statistically significant differences were not reported for any appearance-related outcomes and for only 5 of 25 (20\%) other psychological outcomes. Given heterogeneity of populations and interventions, poor reporting and high risk of bias, quantitative synthesis was not possible. Conclusions Conclusions about effectiveness of nonsurgical cosmetic or other camouflage interventions could not be drawn. Well-designed and conducted trials are needed. Without such evidence, clinicians or other qualified individuals should engage with patients
Strengths and limitations of this study

A peer-reviewed database search strategy was used to ensure rigour and comprehensiveness.

- The Template for Intervention Description and Replication checklist was used to assess completeness of reporting of intervention characteristics.

- A patient advisory team was involved in reviewing and interpreting results and providing suggestions for implications.

- The outcome domains and measures reported by included trials were heterogeneous, which made comparison across trials difficult.

- The low quality of evidence available did not allow conclusions to be drawn about effectiveness.

interested in cosmetic interventions in shared decision making, outlining potential benefits and harms, and the lack of evidence to inform decisions.

PROSPER0 registration number CRD42018103421.

\section{INTRODUCTION}

Visible differences in physical appearance due to medical conditions or injuries are associated with poor social and mental health outcomes. ${ }^{1-3}$ Individuals living with visible appearance differences experience unique social challenges, such as staring and invasive questions, and many experience fear of negative evaluation, social interaction anxiety and social isolation. ${ }^{14}$ Intrapersonal consequences include body image dissatisfaction, low self-esteem and high levels of emotional distress. $^{1-6}$

Interventions that have been used to attempt to improve outcomes among people with visible appearance differences include surgical, ${ }^{67}$ psychological $^{28-10}$ and non-surgical 
cosmetic and other camouflage ${ }^{11} 12$ interventions. Nonsurgical cosmetic and other camouflage interventions are comprised of non-invasive techniques intended to minimise or disguise visible appearance differences. ${ }^{11} 12$ These may include the use of cosmetics (eg, concealer), prosthetics (eg, prosthetic eye), clothing and stylistic accessories (eg, scarves) or hair (eg, wigs). ${ }^{1}$

A 2018 systematic review examined the effects of cosmetic camouflage in children and adults with disfiguring skin disorders on quality of life outcomes. ${ }^{11}$ It included cosmetic interventions but not other types of camouflage interventions, and only included people with skin conditions but not appearance differences that were congenital, acquired from medical conditions, treatments or injuries. Only 6 of 18 included studies were randomised controlled trials (RCTs), and the systematic review did not compare any outcomes between participants randomised to receive camouflage interventions and participants in control arms. Instead, the systematic reported only within-group pre and postmean reductions in Dermatological Life Quality Index (DLQI) scores for the nine studies with pre-post data that reported that measure.

Pre-post analyses of intervention effects, however, are highly prone to bias, and when there is no control group it is not possible to determine with any degree of confidence whether an intervention had an effect or to attempt to quantify effects. This would require a precise understanding of the natural history of symptoms or to be able to confidently assume that symptoms do not change over time without intervention. This is typically not the case, however, in trials of interventions that attempt to improve mental health or other areas of subjective wellbeing. In these areas, participants often seek services and enrol in trials when they are experiencing high levels of symptoms or feeling poorly, and regression to the mean is common. ${ }^{13-17}$ In major depression intervention trials, for instance, approximately $40 \%$ of participants assigned to placebo groups in drug trials or no-treatment groups in psychological intervention trials achieve remission, which has been termed 'spontaneous recovery'. ${ }^{18}$ The Cochrane Handbook discourages inclusion of evidence from non-randomised studies when conducting trials is highly feasible and when evidence from non-randomised trials would be unlikely to provide interpretable results. ${ }^{19}$

We conducted a systematic review with the aim of informing healthcare providers and the public about the effectiveness of cosmetic and other camouflage interventions to improve outcomes among people with visible appearance differences or, in the absence of informative evidence, to identify this shortcoming and describe improvements that are needed. Thus, the objective was to evaluate evidence from RCTs of cosmetic and other camouflage interventions with training or instructions delivered (1) in person or (2) as self-help material (eg, leaflets, internet based) on appearance-related outcomes, general psychological outcomes and adverse effects among adults with appearance differences that were congenital, from injury, a medical condition or medical treatment.

\section{METHODS}

Results were reported per the Preferred Reporting Items for Systematic Reviews and Meta-Analyses statement. ${ }^{20}$

\section{Identification of eligible studies}

Eligible studies were reports of RCTs in any language that examined the effects of non-surgical cosmetic or other camouflage interventions on appearance-related or general psychological outcomes among adults (ages 18 and over) with congenital or acquired visible appearance differences from injury (eg, burn injury), disease or medical condition (eg, scleroderma) or medical treatment (eg, resection of facial tumour, breast reconstruction). Studies that included both children and adults were only included if results for the adults were reported separately or if greater than $80 \%$ of participants were adults. Trials that enrolled only individuals with visible appearance differences due to self-harm were excluded. Eligible interventions were defined as interventions with a core component that involved teaching, modelling or demonstrating the modification or enhancement of some aspect of physical appearance through non-surgical cosmetic or other camouflage strategies. Eligible intervention formats included individual and group formats and delivery via any mode (eg, in-person, web-based, bibliotherapy) by any type of provider. Eligible comparators included (1) any inactive control condition (eg, usual care, attention control, sham procedure); (2) a non-cosmetic intervention designed to target appearance concerns (eg, psychological intervention) and (3) another eligible cosmetic intervention. To be eligible for inclusion, studies must have reported (1) appearance-related psychological outcomes; (2) general psychological outcomes, including symptoms of depression or anxiety or (3) adverse effects from the intervention.

\section{Search strategy}

A health sciences librarian (JB) developed the search strategy and performed the literature searches in MEDLINE (Ovid), EMBASE (Ovid), PsycINFO (Ovid) CINAHL and Cochrane Central from database inception to 24 October 2020 with no language restrictions. The Cochrane Highly Sensitive Search Strategy for identifying randomised trials in MEDLINE: sensitivity-maximising version (2008 revision) was used..$^{21}$ The MEDLINE strategy was developed with input from the project team and peerreviewed by a second librarian using the Peer Review of Electronic Search Strategies guidelines. ${ }^{22}$ After the initial MEDLINE strategy was finalised, it was adapted for use in the other databases. The complete search strategy is available in online supplemental file 1 . We also reviewed reference lists of included articles and relevant reviews and conducted a forward citation search via Google Scholar. ${ }^{23}$ We searched ClinicalTrials.gov, ISRCTN (www. 
isrctn.com) and the WHO registry search portal (apps. who.int/trialsearch) to identify any additional trials that may have been initiated but not published.

Search results were initially downloaded into the citation management database RefWorks (RefWorks, RefWorks-COS, Bethesda, Maryland, USA) for duplicate removal. Unique citation records were then transferred into the systematic review programme DistillerSR (Evidence Partners, Ottawa, Canada). DistillerSR was used to track results of the review process. Two investigators independently reviewed titles and abstracts for eligibility. If either reviewer deemed a citation potentially eligible based on a review of the title and abstract, a fulltext review of the article was completed by two reviewers independently. Any disagreement between reviewers after full-text review was resolved by consensus, including consultation with an independent third reviewer if necessary.

\section{Data extraction and analysis}

Data were extracted from included RCTs by two investigators using a standardised electronic data collection form in DistillerSR. Disagreements were resolved by consensus and, if necessary, consultation with an independent third reviewer. See online supplemental file 2 for a complete list of extracted variables.

We used the Template for Intervention Description and Replication (TIDieR) checklist ${ }^{24}$ to evaluate the degree to which interventions were reported sufficiently to allow replication in research or practice. The checklist is comprised of 12 items that assess reporting of the intervention name; the rationale or theory underlying the intervention; physical or informational material used; procedures and processes of the intervention; the intervention provider and their background; the mode of delivery, such as group or face to face; the location where the intervention was delivered and necessary infrastructure; the number of sessions, schedule and duration; if any tailoring was done and how; any modifications made to the intervention; if adherence or fidelity was assessed and how; and, if adherence or fidelity was assessed, the extent to which the intervention was delivered as planned.

We used the 2011 version of the Cochrane Collaboration's risk of bias tool to evaluate risk of bias. ${ }^{25}$ The tool has seven domains, including random sequence generation, allocation concealment, blinding of participants and personnel, blinding of outcome assessment, incomplete outcome data, selective outcome reporting and any other potential sources of bias. Studies were rated low, unclear or high risk of bias on each domain.

We did not pool results across trials because of the heterogeneity of interventions and trial designs and concerns about risk of bias. Rather, we descriptively reported trial characteristics and outcomes. Between groups standardised mean differences (SMDs) were calculated for each relevant outcome using Hedges's $g^{26}$ when possible. For trials where sufficient data were not provided to calculate SMDs, other relevant information that could be used to describe intervention outcomes (eg, median change in each trial arm with $\mathrm{p}$ value) was reported.

\section{Patient and public involvement}

We assembled a Body Image Patient Advisory Team comprised of four female and one male persons with scleroderma, a rare disease that often causes changes in appearance, to advise on the present systematic review. The advisory team participated in a patient panel to discuss the study objectives, findings and implications of the study. Members of the patient panel were also sent copies of the present manuscript prior to submission and asked to provide any feedback or suggestions. Members of the advisory team commented on issues related to characteristics of participants in the trials we reviewed, the acceptability of camouflage interventions and delivery of these interventions via healthcare providers. These comments were incorporated into the Discussion section of the manuscript.

\section{Modifications to protocol}

When we developed the review protocol, we did not anticipate that we might encounter trials that enrolled only individuals with visible appearance differences from selfinflicted injuries, and eligibility criteria did not address this group. We subsequently added visible appearance differences from self-injury as an exclusion criterion because we deemed the population to be substantively different than the target population; interventions for this population would need to address factors related to self-harming behaviour, in addition to appearance, which would be out of scope for our intended target population.

\section{RESULTS}

\section{Search results}

The combined database searches identified 1085 unique titles and abstracts. Of these, 1062 were excluded after screening titles and abstracts and 19 after full-text review, resulting in 4 eligible RCTs. One additional eligible RCT ${ }^{27}$ was identified via a database search that we conducted for a separate systematic review on psychological interventions (PROSPERO: CRD42018100632), and a second additional eligible $\mathrm{RCT}^{28}$ was identified via manual searching of a relevant systematic review, ${ }^{11}$ resulting in six included $\mathrm{RCTs}^{27-32}$ (see figure 1). No additional eligible trials were identified via manual searching of reference lists of included articles, citation tracking or review of trial registries. One ongoing trial was identified via the review of trial registries (NCT03540966), but results were not available as of 2 November 2020.

\section{Characteristics of included studies}

The six included RCTs were published between 2006 and $2019{ }^{27-32}$ Five trials compared an intervention to a no-intervention, waitlist or usual care control, ${ }^{27}{ }^{29-32}$ and one 


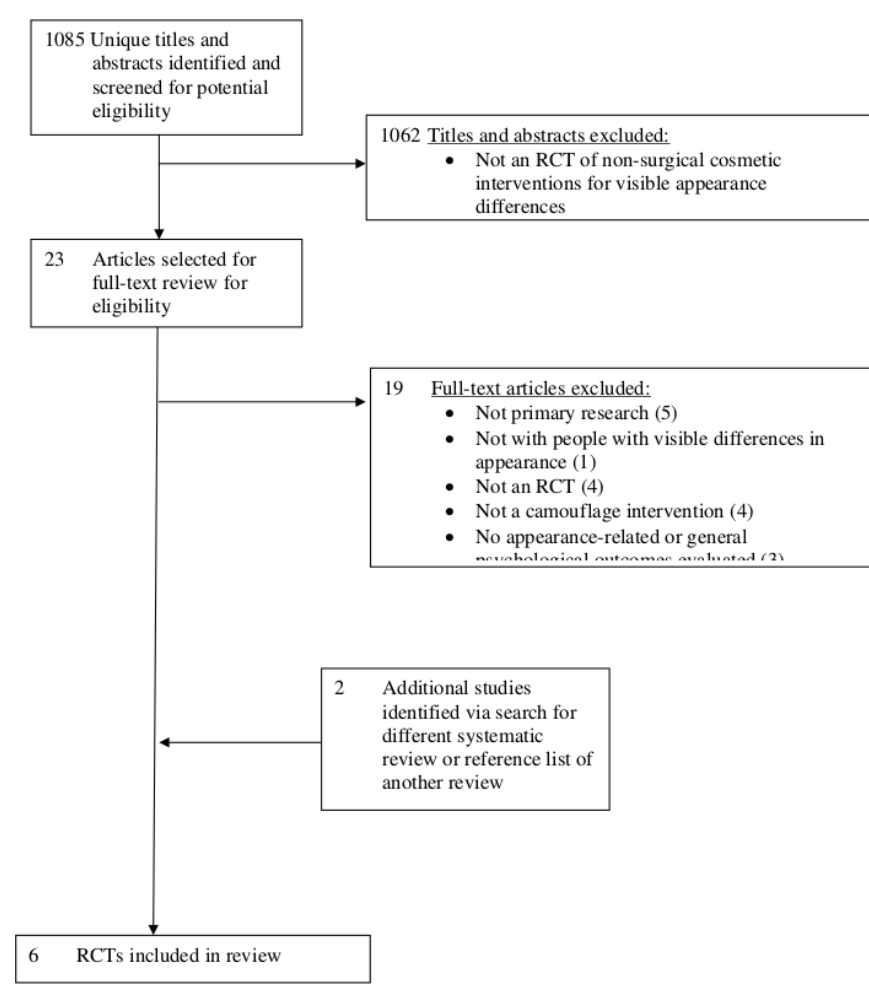

Figure 1 PRISMA flow diagram of study selection. PRISMA, Preferred Reporting Items for Systematic Reviews and MetaAnalyses; RCTs, randomised controlled trials.

trial $^{28}$ was a head-to-head comparison of two different camouflage products.

\section{Comparison to no intervention or usual care}

Trial participants included survivors of gynaecologic malignancies with chemotherapy-induced alopecia, ${ }^{27}$ head and neck cancer survivors, ${ }^{29}$ people living with acne vulgaris, ${ }^{30}$ head and neck skin cancer survivors ${ }^{31}$ and survivors of breast cancer. ${ }^{32}$ The number of participants per trial ranged from 24 to 136 (mean: 65; median: 50), and there was a total of 324 participants, with 162 participants each in intervention and control arms of trials. All participants were female. The mean ages for participants in each trial ranged from 29 to 67 (see table 1).

The trials were conducted in the USA, ${ }^{27}$ Taiwan, ${ }^{29}$ Japan, ${ }^{30}$ Italy $^{31}$ and Germany. ${ }^{32}$ The interventions ranged from one to four sessions. Formats included videotape review, ${ }^{27}$ combined individual and group sessions, ${ }^{29}$ combined individual sessions and videotape, ${ }^{30}$ and group sessions. $^{31} 32$ Two RCTs required either self-report or investigator confirmation of appearance changes from an illness, injury or medical procedure as a condition of eligibility $^{2932}$; none, though, set a threshold for severity. No trials included patient report of concern about appearance as part of eligibility criteria, even though the trials targeted appearance-related and psychological variables.

In two RCTs, participants were provided with cosmetic camouflage products. ${ }^{30} 31$ In one trial, ${ }^{30}$ which included people with acne, all products were developed and provided by Nov Pharmaceuticals and included skincare and cosmetic products and instructions on using the products that were specific to people with acne. In the second trial, customised camouflage plans that were first submitted to participants for approval were provided, and cosmetic camouflage products were provided by Istituto Ganassini $^{31}$; however, the specific cosmetic products were not described. Three of the trials ${ }^{2729}$ provided instructions on using camouflage techniques but did not provide specific products. In one trial, ${ }^{27}$ participants viewed a $45 \mathrm{~min}$ video that provided camouflage suggestions and techniques, including information on cosmetic camouflage and wigs. In another, hands-on instruction and practice with cosmetic camouflage was initiated, and a 25-page manual was provided. ${ }^{29}$ In another, a group makeup workshop was provided and followed with a professional photoshoot and delivery of edited photographs. ${ }^{32}$

Comparators included standard counselling on hair loss, ${ }^{27}$ unspecified routine care ${ }^{29}{ }^{31}$ conventional acne treatment $^{30}$ and a wait-list control. ${ }^{32}$ Four trials included appearance-related outcome measures, ${ }^{27} 293132$ two trials included a depression symptom outcome measure, ${ }^{29} 32$ one trial included an anxiety outcome measure ${ }^{29}$ and five trials included general mental health outcome measures. $^{27}{ }^{29-32}$ No trials reported on adverse effects from participation.

\section{Head-to-head Trial}

The head-to-head trial, ${ }^{28}$ which was conducted in France, included 88 healthy female volunteers with various facial aesthetic imperfections (eg, melisma, scars from chicken pox), the presence of which were validated by clinical examination. The mean age of participants was 30 . Participants were randomised to receive either a heaviercoverage liquid or a lighter-coverage powder product; all participants received recommendations and instructions from a professional makeup trainer in a single individual session. The study outcome was a body image-specific quality of life measure (see table 1 ).

\section{Synthesis of outcomes}

Comparison to no intervention or routine care controlled trials

There was sufficient information to calculate intervention effect sizes (SMDs) and confidence intervals for two of the five trials that compared an intervention group to a no intervention, wait list or routine care control group, ${ }^{29}{ }^{30}$ only one of which ${ }^{29}$ included an appearancerelated outcome.

Appearance-related outcomes: In the four trials that reported appearance-related outcomes, ${ }^{27} 293132$ there were no statistically significant differences between camouflage intervention and control groups. Reported effect sizes were generally negligible (see table 2).

General psychological symptoms: Across a total of 25 general psychological outcome measurement points reported in the five trials, five $(20 \%)$ were statistically significant. There was a small to moderate effect on the Leibowitz Social Anxiety Scale-Anxiety subscale at 8 weeks (SMD: -0.41 CI: -0.89 to -0.07 ) in a trial that randomised 


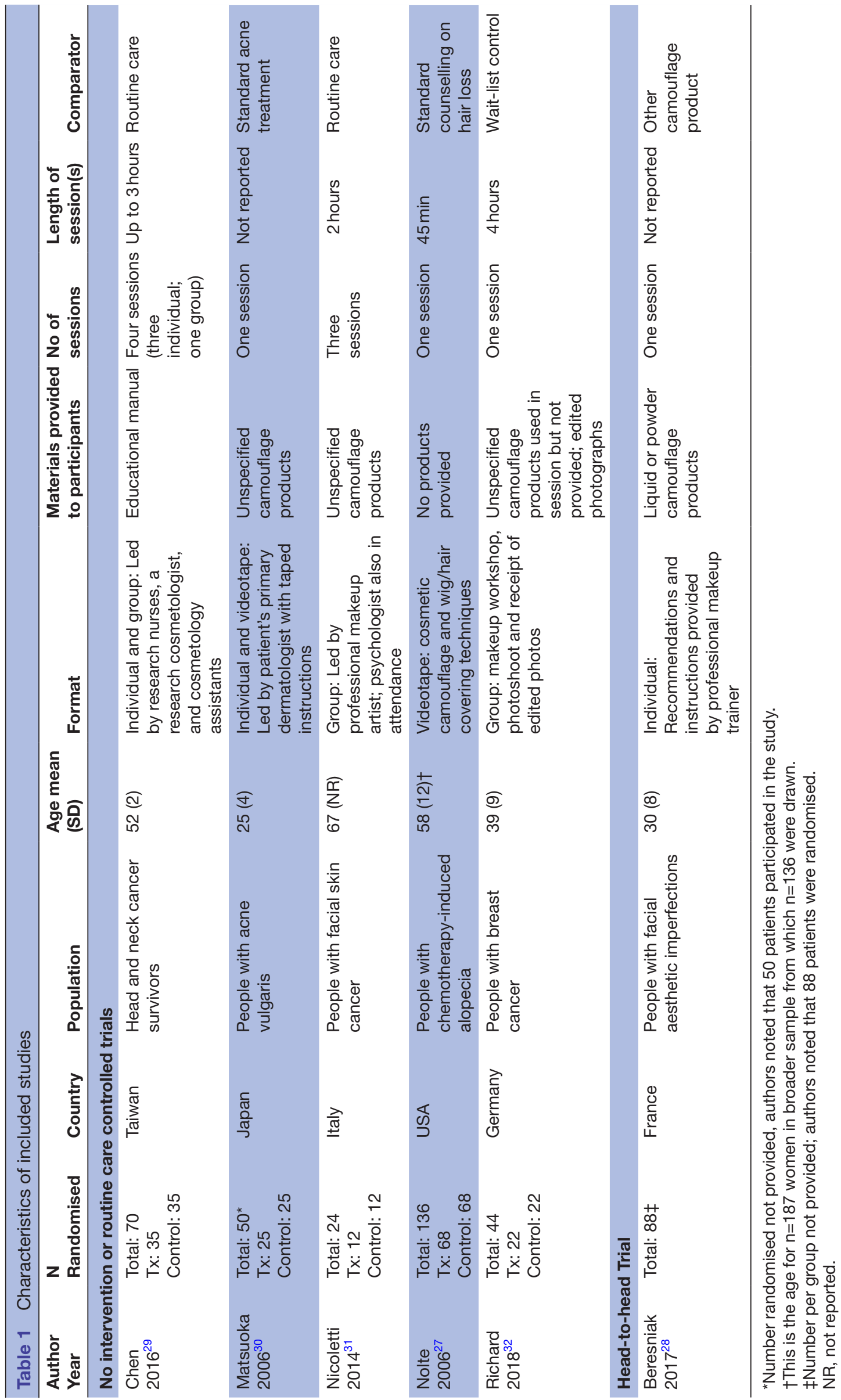

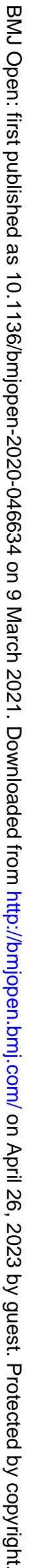


Table 2 Outcomes of included trials

\begin{tabular}{|c|c|c|c|c|c|}
\hline Study & $\mathrm{N}$ randomised & Appearance related & General mental health & Depression & Social anxiety \\
\hline \multicolumn{6}{|c|}{ No intervention or routine care controlled trials } \\
\hline $\begin{array}{l}\text { Chen } \\
2016^{29}\end{array}$ & $\begin{array}{l}\text { Total: } 70^{\star} \\
\text { Tx: } 35 \\
\text { Control: } 35\end{array}$ & $\begin{array}{l}\text { BIS: SMD }(95 \% \mathrm{Cl}) \\
4 \text { weeks } \\
-0.07(-0.54 \text { to } 0.40) \\
8 \text { weeks } \\
-0.08(-0.56 \text { to } 0.39) \\
12 \text { weeks } \\
-0.43(-0.92 \text { to } 0.06)\end{array}$ & $\begin{array}{l}\text { RSES: SMD }(95 \% \mathrm{Cl}) \\
4 \text { weeks } \\
-0.19(-0.67 \text { to } 0.28) \\
8 \text { weeks } \\
0.31(-0.17 \text { to } 0.79) \\
12 \text { weeks } \\
-0.03(-0.52 \text { to } 0.45)\end{array}$ & $\begin{array}{l}\text { HADS-D: SMD } \\
(95 \% \mathrm{Cl}) \\
4 \text { weeks } \\
0.33(-0.15 \text { to } 0.80) \\
8 \text { weeks } \\
-0.08(-0.56 \text { to } 0.39) \\
12 \text { weeks } \\
-0.14(-0.62 \text { to } 0.35)\end{array}$ & 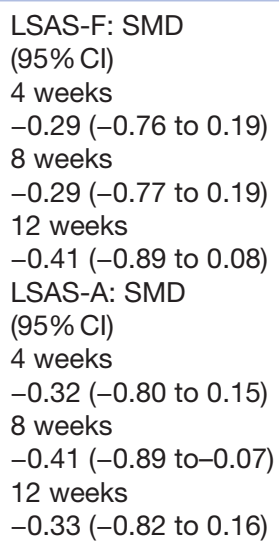 \\
\hline $\begin{array}{l}\text { Nicoletti } \\
2014 \ddagger^{31}\end{array}$ & $\begin{array}{l}\text { Total: } 24 \S \\
\text { Treatment: } 12 \\
\text { Control: } 12\end{array}$ & $\begin{array}{l}\text { BUT-GSI: } \\
3 \text { months } \rrbracket: \\
\text { Tx: change }=-0.26, \\
\text { IQR }=-0.72,0.16 \\
\text { Control: change }=0.03 \text {, } \\
\text { IQR }=-0.08,0.34 \\
\mathrm{p}=0.371 \\
6 \text { months } \rrbracket: \\
\text { Tx: change }=-0.15 \\
\text { IQR }=-0.54,0.45 \\
\text { Control: change }=0.12, \\
\text { IQR }=-0.09,0.64 \\
p=0.318\end{array}$ & 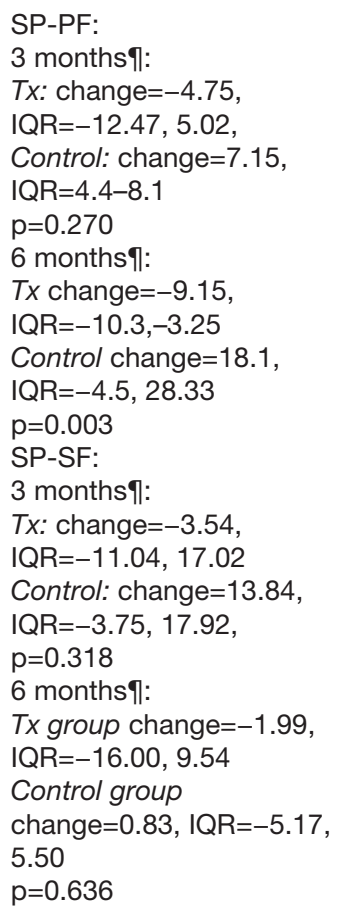 & NA & NA \\
\hline $\begin{array}{l}\text { Nolte } \\
2006^{\star * 27}\end{array}$ & $\begin{array}{l}\text { Total: } 136 \\
\text { Treatment: } 68 \\
\text { Control: } 68+\dagger\end{array}$ & $\begin{array}{l}\text { BCSCS-BC: } \\
\text { Post-tx: } \\
\text { F(1,132)=0.11, } p=0.74 \\
\text { (Tx: 68; Control 68) }\end{array}$ & $\begin{array}{l}\text { BCSCS-SC: } \\
\text { Post-tx } \\
F(1,132)=0.32, p=0.57 \\
(T x: 68 ; \text { Control 68) }\end{array}$ & NA & NA \\
\hline
\end{tabular}


Table 2 Continued

\begin{tabular}{|c|c|c|c|c|c|}
\hline Study & $\mathbf{N}$ randomised & Appearance related & General mental health & Depression & Social anxiety \\
\hline $\begin{array}{l}\text { Richard } \\
2018^{32}\end{array}$ & $\begin{array}{l}\text { Total: } 44 \\
\text { Treatment: } 22 \\
\text { Control: } 22 \ddagger \ddagger\end{array}$ & $\begin{array}{l}\text { BIS: } \\
2 \text { weeks, } 4 \text { weeks: NR; } \\
\text { Improvements in body } \\
\text { image were independent of } \\
\text { treatment }(p>0.05)\end{array}$ & $\begin{array}{l}\text { RSES: } \\
2 \text { weeks: } d=-0.68 \S \S \\
4 \text { weeks: } d=-0.72 \S \S\end{array}$ & $\begin{array}{l}\text { CES-D: } \\
2 \text { weeks: } d=-0.83 \S \S \\
4 \text { weeks: } d=-0.45 \S \S\end{array}$ & NA \\
\hline
\end{tabular}

Head-to-head trial

$\begin{array}{ll}\text { Beresniak } & \text { Total: 88ा } \\ 2017^{28} & \end{array}$

treatment $(p>0.05)$

$2017^{28}$

BeautyQOL-Attractiveness:
at 1 and 3 weeks, mean

NA

NA

$A>$ mean $B ; p>0.05$

BeautyQOL-Social Life: at 1

and 3 weeks, mean $A>$ mean

B; $p>0.05$

BeautyQOL-Mood: at 1 and

3 weeks, mean $A>$ mean $B$;

$\mathrm{p}>0.05$

BeautyQOL-Self Confidence:

1 week: mean $A>$ mean $B$;

$p<0.05$

3 weeks: mean $A>$ mean $B$;

$p>0.05$

Scores reversed for some outcome measures such that higher scores indicate worse functioning for all measures and negative between-group effects reflect better outcomes in the intervention groups.

${ }^{*} \mathrm{Tx}(\mathrm{n}: 35,34,32)$ and control (n: 34, 34, 34) at 4, 8 and 12 weeks, respectively.

†Number randomised not provided, noted that 50 patients participated in the study.

†Effect sizes could not be calculated.

$\S T x(n: 12,12)$ and control $(n: 12,12)$ at 3 and 6 months, respectively.

IData reported as change score (IQR) from baseline to 3 months and $p$ value is for between-group difference.

**Intervention effect comparing baseline scores to scores after course 4 of chemotherapy using BCSCS scores during course 3 of chemotherapy as a covariate reported.

††Tx (n: 68) and control (n: 68) at post-tx measurement.

打 (n: 20, 29) and control (n: 19, 19) at 2 and 4 weeks.

$\S \S B$ Baseline corrected.

१ीTx and Control $n$ not provided; 86 participants completed study.

BCSCS-BC, Body Cathexis/Self-Cathexis Scale-Body Cathexis; BCSCS-SC, Body Cathexis/Self-Cathexis Scale-Self-Cathexis; BIS, Body Image Scale; BUT-GSI, Body Uneasiness Test Global Score Index; DLQI-PR, Dermatology Life Quality Index-Personal Relationships; DLQI-SF, Dermatology Life Quality Index-Symptoms/Feelings; HADS-D, Hospital Anxiety and Depression Scale; LSAS-A, Liebowitz Social Anxiety Scale-Anxiety; LSAS-F, Liebowitz Social Anxiety Scale-Fear; NA, not available; RSES, Rosenberg Self-Esteem Scale; SMD, standardised mean difference; SP-PF, Satisfaction Profile-Psychological Functionality; SP-SF, Satisfaction Profile-Social Functionality; Tx, treatment/intervention group; WHOQOL-26-P, WHO Quality of Life-Psychological domain; WHOQOL-26-SR, WHO Quality of Life-Social Relationships domain.

70 head and neck cancer survivors to individual and group sessions or routine care, ${ }^{29}$ although the effect was smaller and not statistically significant at 4 or 12 weeks, and the effect for the Fear subscale was non-significant at all three time points. There were moderate to large magnitude effects for depressive symptoms (d: -0.83 at 2 weeks and d: -0.43 at 4 weeks) and self-esteem (d: 0.68 at 2 weeks and d: 0.72 at 4 weeks) in a trial that randomised 44 breast cancer patients to a single group session versus wait-list control, though it was not clear if they were statistically significant ${ }^{32}$ (see table 2 ).

\section{Head-to-head trial}

In the head-to-head trial, which compared liquid and powder camouflage products among 88 people with a variety of aesthetic imperfections, ${ }^{28}$ sufficient information to calculate effect sizes was not provided. Of the four outcomes measured at two time points (eight outcomes total), one was reported to be statistically significantly different between groups (a self-confidence subscale at 1 week).

\section{Reporting of intervention components and risk of bias}

As assessed using the TIDieR checklist, few trial reports described the interventions used with sufficient detail to fully understand what had occurred or to consider replication (see table 3 ). Materials were not sufficiently reported in terms of describing specific materials used or providing details on how to access the materials for all of the trials. ${ }^{27-32}$ Although all of the studies described who provided the intervention, four did not provide details about training or qualifications of the interventionists specific to camouflage or visible appearance differences. ${ }^{28-32}$ Adherence was not assessed in any of the six trials.

Risk of bias was evaluated using the Cochrane criteria with most studies rated as high or unclear across the domains assessed (see table 4). Random sequence generation procedures were only described in two of the studies. ${ }^{29}{ }^{31}$ Due to the nature of the interventions and self-reported psychological outcomes used in the studies, risk of bias due to a lack of blinding of participants/ 


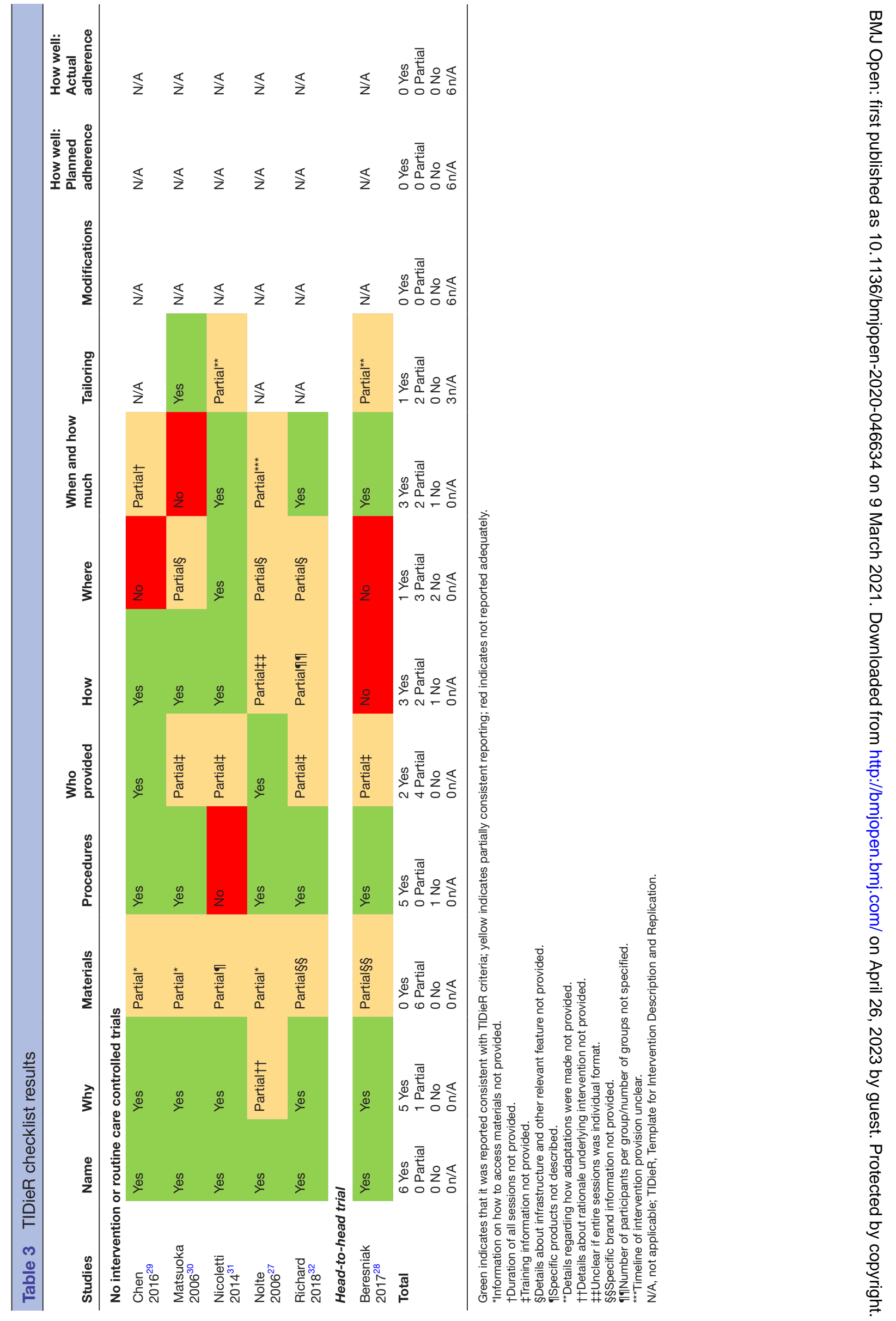




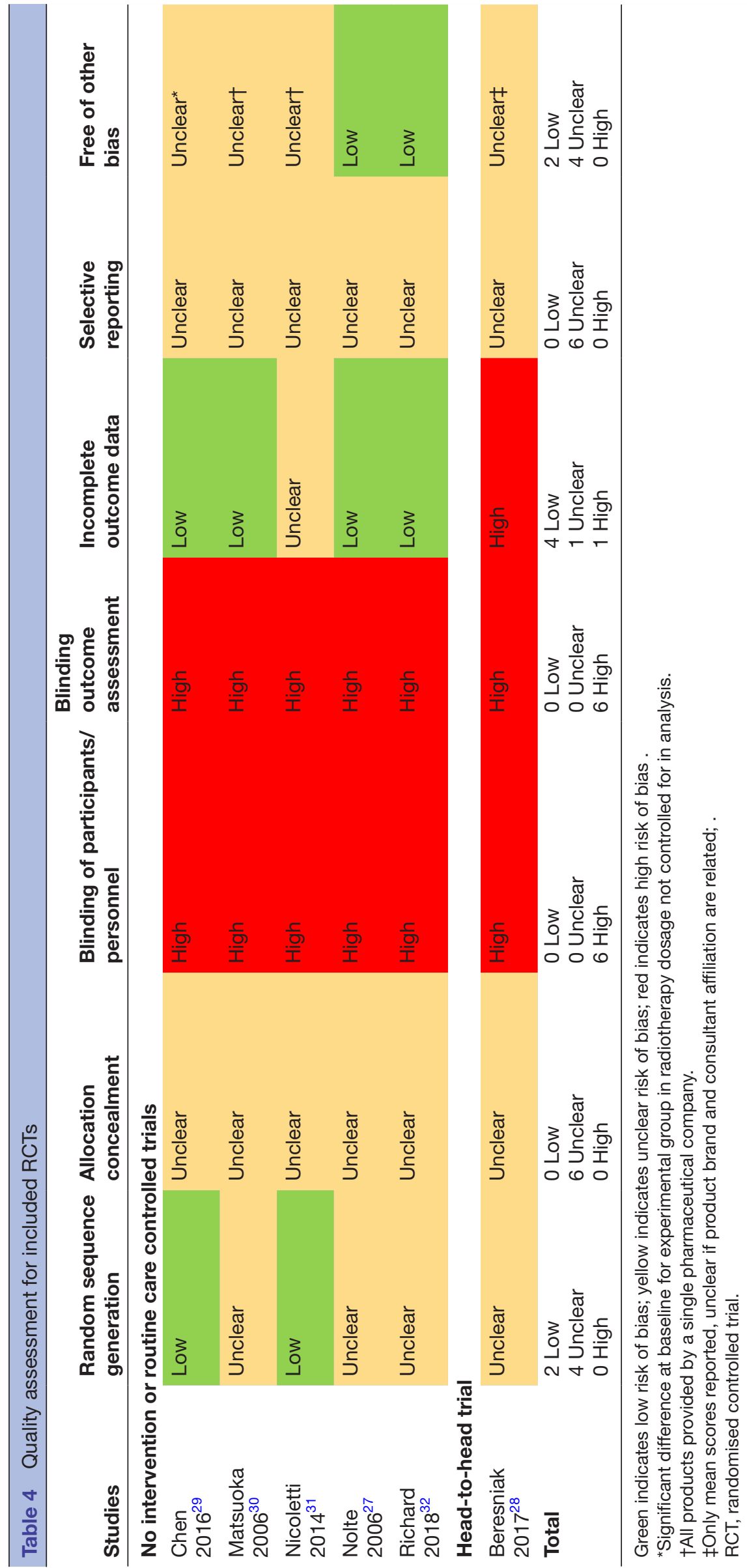

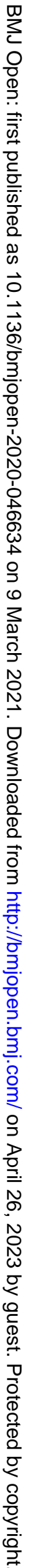


personnel and outcome assessment was high across all studies. Overall, of the seven domains assessed (including 'other'), three RCTs were rated as unclear or high in five domains, ${ }^{272932}$ two in six domains ${ }^{3031}$ and one in all seven domains. ${ }^{28}$

\section{DISCUSSION}

\section{Main findings}

We identified five RCTs that compared camouflage interventions to no treatment, waiting list, or routine care controls, and one head-to head trial that compared two camouflage products. Trials included participants from populations with a range of visible appearance differences, including people with head and neck cancer, acne vulgaris, facial skin cancer, chemotherapy-induced alopecia, breast cancer and non-defined facial aesthetic imperfections. Risk of bias was unclear or high in at least five of seven possible domains for all included RCTs. Furthermore, outcomes were incompletely reported in many trials, and we could only calculate effect sizes for a small proportion of outcomes that were described. Very few statistically significant results were identified. Additionally, based on TIDieR checklist ratings, interventions were generally not described with enough information to support replication or use in practice. Given these limitations, a meaningful quantitative synthesis of the findings from included RCTs was not possible, and conclusions about the likely effectiveness of non-surgical cosmetic interventions on appearance-related or general psychological outcomes could not be drawn with any degree of confidence. No trials reported on adverse effects.

\section{Findings in context}

A previous review ${ }^{11}$ used preintervention and postintervention scores among only studies that used a single quality of life measure, the DLQI ${ }^{33}$ and concluded that reductions in scores across nine studies of varying designs suggested effectiveness of cosmetic camouflage on quality of life outcomes. That review did not attempt to synthesise between-group effects or describe any limitations due to not evaluating between-group effects. There are several reasons that help explain why our conclusion differs from the conclusion of that review. First, the specific focus of the previous review was the role of cosmetic camouflage on quality of life outcomes, whereas the present review was broader in scope and included any non-surgical cosmetic intervention (eg, wigs, prosthetics) and any appearance-related and general psychological outcomes. Second, the previous review included studies of children and adults, whereas our review was limited to adult samples. Third, and most importantly, the conclusions of the previous review were drawn from precomparisons and postcomparisons without control groups, whereas we included only RCTs. RCTs are considered the goldstandard study design for establishing the effectiveness of psychological interventions and inferring causation. ${ }^{34}$ There is a high risk in trials of psychosocial interventions, in which people often consent to participate when they are struggling with concerns, that the natural trajectory of included participants may be to improve over time. Non-randomised trials in this area are pretrials and posttrials that must assume that there would be no change over time without the intervention. Systematic reviews that draw conclusions and make recommendations from these kinds of trials are at high risk of misleading clinicians who seek to provide the best possible care to people with visible differences in appearance, misleading people with visible appearance differences about their options, and losing the opportunity to encourage researchers to engage in improved trials that answer a critical healthcare question.

\section{Limitations}

Several important methodological limitations were noted in the RCTs included in the present review, and there are implications for future research. First, study aims were often ambiguous, and it was unclear if what was being tested was a specific product, camouflage in general or instructions on using camouflage. Second, few trials provided information that would be needed for replication or use in practice. Third, no studies had eligibility criteria for baseline appearance concerns or subjective severity. Only three of the trials ${ }^{28} 29$ included eligibility criteria relating to confirming the presence of appearance differences; however, no trials included a threshold for objective severity or required that participants had appearance-related concerns. Thus, some participants may have been enrolled even though they did not have appearance concerns, and those participants would not have been able to benefit from the interventions. Fourth, none of the studies reported on harms from the interventions. It is possible that some products may exacerbate skin conditions and lead to worse objective appearance or psychological outcomes. Fifth, no studies included men, limiting generalisability of the findings. This was a limitation that was emphasised by the Patient Advisory Team, which raised the concern that the acceptability of camouflage among men is not known. Sixth, the small number of RCTs available, the generally small sample sizes included and the poor reporting of trial methods and results limited utility of the trials and the ability to draw conclusions from what they reported; these are common issues in rare disease and visible appearance differences research. ${ }^{35}$

The findings of this review should be interpreted in the context of several limitations of the review itself. First, although a systematic search designed by a medical librarian was used, incompleteness of the evidence reported and publication bias limiting null results are concerns for any systematic review. Second, the small number of studies eligible for inclusion in the review and differences in how data were reported did not allow for publication bias to be assessed. Third, given the low quality of trial evidence available, in addition to not being able to draw conclusions about overall effectiveness, examination 
of potentially relevant participant (eg, age, sex, marital status) and condition (eg, years of living with the visible appearance difference, patient-perceived and observerrated severity) variables could not be undertaken. Fourth, even with better quality evidence, the inconsistency across studies in outcome domains and measures used to evaluate effects would make drawing conclusions difficult. Ideally, a core set of outcome domains and measures would be used consistently.

\section{Implications of findings}

There are clinical implications of this review. Because of high patient interest in non-surgical cosmetic treatments ${ }^{12}$ and the fact that for some conditions causing visible appearance differences non-surgical cosmetic treatments are the only treatment option available, many people may consider using camouflage or other cosmetic interventions. A concern raised by members of our Body Image Patient Advisory Team, however, was that it may be better to accept one's visible appearance differences rather than engaging in behaviours to attempt to hide or conceal them. It was noted that suggesting camouflage may send an undesired message to patients who are comfortable with their appearance or who are working to be more comfortable. In addition to improving the quality and reporting of trials, future researchers may consider the role of internet delivery of interventions, as health interventions are increasingly delivered via the internet and have been shown to be similarly effective to face-to-face interventions. ${ }^{36-38}$ Online delivery could enable wider recruiting and larger sample sizes as well as more efficient dissemination if an intervention is found to be effective.

Patients who are interested in these approaches and may wish to discuss advantages and disadvantages with healthcare providers or other qualified individuals. Given the lack of high-quality evidence, clinicians are left with a gap not only around whether interventions are likely to be effective, but whether there may be adverse effects to consider. In light of these limitations, we recommend that clinicians working with visible appearance differences populations engage in a shared decision-making approach with patients who express an interest in or who may benefit from non-surgical cosmetic intervention, ${ }^{39}$ such that education is provided on the current lack of empirical information about clinical and psychological harms as well as effectiveness of interventions.

\section{CONCLUSION}

In summary, the present review did not find evidence to evaluate benefits or harms of the use of non-surgical cosmetic treatments in visible appearance differences. There are several recommendations for future RCTs evaluating the efficacy of non-surgical cosmetic interventions in visible appearance differences populations, such as the inclusion of appearance-related eligibility criteria and the use of internet-based and other feasible delivery modalities. Until more robust scientific evidence becomes available, clinicians can engage in shared decision making with patients to determine whether engaging in non-surgical cosmetic interventions given the current lack of empirical support may be warranted.

\section{Author affiliations}

${ }^{1}$ Lady Davis Institute for Medical Research, Jewish General Hospital, Montreal, Quebec, Canada

${ }^{2}$ Department of Psychiatry, McGill University, Montreal, Québec, Canada ${ }^{3}$ Department of Psychology, San Diego State University, San Diego, California, USA

${ }^{4}$ Department of Psychology, McGill University, Montreal, Québec, Canada

${ }^{5}$ Department of Clinical Psychology, Behavioural Science Institute, Radboud University, Nijmegen, The Netherlands

${ }^{6}$ Schulich Library of Physical Sciences, Life Sciences, and Engineering, McGill University, Montreal, Québec, Canada

${ }^{7}$ Joint Doctoral Program in Clinical Psychology, San Diego State University/ University of California San Diego, San Diego, California, USA

${ }^{8}$ Department of Epidemiology, Biostatistics, and Occupational Health, McGill University, Montreal, Québec, Canada

${ }^{9}$ Department of Medicine, McGill University, Montréal, Québec, Canada

${ }^{10}$ Department of Educational and Counselling Psychology, McGill University,

Montréal, Québec, Canada

${ }^{11}$ Biomedical Ethics Unit, McGill University, Montreal, Québec, Canada

Collaborators SPIN Body Image Patient Advisory Team Members: Amy Gietzen, Scleroderma Foundation, Tri-State Chapter, Binghamton, New York, USA; Karen Gottesman, Scleroderma Foundation, Los Angeles, California, USA; Genevieve Guillot; Sclérodermie Québec, Montreal, Quebec, Canada; Nancy Lewis, Ontario, Canada; Joep Welling, NVLE Dutch patient organization for systemic autoimmune diseases, Utrecht, The Netherlands.

Contributors SG, DBR, AC-J and BDT were responsible for the study concept and design, drafted the study protocol, contributed to data extraction, contributed to drafting the manuscript and approved the final manuscript. JB designed and conducted the search strategies, contributed to drafting the manuscript and approved the final manuscript. VLM and LK were responsible for the study concept and design, drafted the study protocol, contributed to drafting the manuscript, and approved the final manuscript. BDT is the guarantor.

Funding The study was supported by funding from the Canadian Institutes for Health Research (CIHR; CMA - 151728). DBR was supported by a CIHR - Health Systems Impact Doctoral Fellowship. AC-J was supported by a CIHR Frederick Banting and Charles Best Canada Graduate Scholarship. BDT was supported by a Tier 1 Canada Research Chair.

Competing interests None declared.

Patient consent for publication Not required.

Provenance and peer review Not commissioned; externally peer reviewed.

Data availability statement All data relevant to the study are included in the article or uploaded as online supplemental information. All data extracted and analysed for the present study are reported in the manuscript and its tables.

Supplemental material This content has been supplied by the author(s). It has not been vetted by BMJ Publishing Group Limited (BMJ) and may not have been peer-reviewed. Any opinions or recommendations discussed are solely those of the author(s) and are not endorsed by BMJ. BMJ disclaims all liability and responsibility arising from any reliance placed on the content. Where the content includes any translated material, BMJ does not warrant the accuracy and reliability of the translations (including but not limited to local regulations, clinical guidelines, terminology, drug names and drug dosages), and is not responsible for any error and/or omissions arising from translation and adaptation or otherwise.

Open access This is an open access article distributed in accordance with the Creative Commons Attribution Non Commercial (CC BY-NC 4.0) license, which permits others to distribute, remix, adapt, build upon this work non-commercially, and license their derivative works on different terms, provided the original work is properly cited, appropriate credit is given, any changes made indicated, and the use is non-commercial. See: http://creativecommons.org/licenses/by-nc/4.0/. 


\section{ORCID iDs}

Danielle B Rice http://orcid.org/0000-0001-5615-7005

Brett D Thombs http://orcid.org/0000-0002-5644-8432

\section{REFERENCES}

1 Rumsey N, Harcourt D. Body image and disfigurement: issues and interventions. Body Image 2004;1:83-97.

2 Bessell A, Moss TP. Evaluating the effectiveness of psychosocial interventions for individuals with visible differences: a systematic review of the empirical literature. Body Image 2007;4:227-38.

3 Thompson A, Kent G. Adjusting to disfigurement: processes involved in dealing with being visibly different. Clin Psychol Rev 2001;21:663-82.

4 Rumsey N, Clarke A, Musa M. Altered body image: the psychosocial needs of patients. Br J Community Nurs 2002;7:563-6.

5 Rumsey N. Body image \& congenital conditions with visible differences. In: Cash TF, Pruzinsky T, eds. Body image: a Handbook of theory, research, and clinical practice. New York: Guilford, 2002: 226-33.

6 Sarwer DB. Cosmetic surgery and changes in body image. In: Cash TF, Pruzinsky T, eds. Body image: a Handbook of theory, research, and clinical practice. New York: Guilford, 2002: 422-30.

7 Pruzinsky T. Body image adaptation to reconstructive surgery for acquired disfigurement. In: Cash TF, Pruzinsky T, eds. Body image: a Handbook of theory, research, and clinical practice. New York: Guilford, 2002: 440-9.

8 Muftin Z, Thompson AR. A systematic review of self-help for disfigurement: effectiveness, usability, and acceptability. Body Image 2013;10:442-50.

9 Norman A, Moss TP. Psychosocial interventions for adults with visible differences: a systematic review. PeerJ 2015;3:e870.

10 et alGholizadeh S, Rice D, Carboni-Jiménez A. The effectiveness of psychological interventions to improve appearance-related and psychological outcomes among adults with visible differences: a systematic review. Prospero CRD42018100632. Available: https:// www.crd.york.ac.uk/prospero/display_record.php?RecordID=100632 [Accessed 02 Nov 2020].

11 Kornhaber R, Visentin D, Thapa DK, et al. Cosmetic camouflage improves quality of life among patients with skin disfigurement: a systematic review. Body Image 2018;27:98-108.

12 Levy LL, Emer JJ. Emotional benefit of cosmetic camouflage in the treatment of facial skin conditions: personal experience and review. Clin Cosmet Investig Dermatol 2012;5:173-82.

13 Balshem H, Helfand M, Schünemann HJ, et al. Grade guidelines: 3. rating the quality of evidence. J Clin Epidemiol 2011;64:401-6.

14 Berkman ND, Lohr KN, Ansari MT, et al. Grading the strength of a body of evidence when assessing health care interventions: an EPC update. J Clin Epidemiol 2015;68:1312-24.

15 Jüni P, Altman DG, Egger M. Systematic reviews in health care: assessing the quality of controlled clinical trials. BMJ 2001;323:42-6.

16 Moher D, Hopewell S, Schulz KF, et al. Consort 2010 explanation and elaboration: updated guidelines for reporting parallel group randomised trials. BMJ 2010;340:c869.

17 Hernán MA, Robins JM. Causal inference. Boca Raton, FL: Chapman \& Hall/CRC, 2018.

18 Cuijpers P. The challenges of improving treatments for depression. JAMA 2018:320:2529-30.

19 Reeves BC, Deeks JJ, Higgins JPT. Chapter 24: Including nonrandomized studies on intervention effects. In: Higgins JPT, Thomas J, Chandler J, eds. Cochrane Handbook for systematic reviews of interventions version 6.1. Cochrane, 2020. www.training.cochrane. org/handbook
20 Moher D, Liberati A, Tetzlaff J, et al. Preferred reporting items for systematic reviews and meta-analyses: the PRISMA statement. Ann Intern Med 2009;151:264-9.

21 Lefebvre C, Glanville J, Briscoe S. Technical Supplement to Chapter 4: Searching for and selecting studies. In: Higgins JPT, Thomas J, Chandler J, eds. Cochrane Handbook for systematic reviews of interventions version 6. Cochrane, 2019. http://training.cochrane.org/ handbook

22 McGowan J, Sampson M, Salzwedel DM, et al. PRESS Peer Review of Electronic Search Strategies: 2015 Guideline Statement. J Clin Epidemiol 2016;75:40-6.

23 Bakkalbasi N, Bauer K, Glover J, et al. Three options for citation tracking: Google Scholar, Scopus and web of science. Biomed Digit Libr 2006;3:7

24 Hoffmann TC, Glasziou PP, Boutron I, et al. Better reporting of interventions: template for intervention description and replication (TIDieR) checklist and guide. BMJ 2014;348:g1687.

25 Higgins JPT, Altman DG, Gøtzsche PC, et al. The Cochrane collaboration's tool for assessing risk of bias in randomised trials. BMJ 2011;343:d5928.

26 Hedges LV. Estimation of effect size from a series of independent experiments. Psychol Bull 1982;92:490-9.

27 Nolte S, Donnelly J, Kelly S, et al. A randomized clinical trial of a videotape intervention for women with chemotherapy-induced alopecia: a gynecologic Oncology Group study. Oncol Nurs Forum 2006;33:305-11.

28 Beresniak A, Auray JP, Duru G. Impact on the quality of life of two cosmetic camouflage products for facial aesthetic imperfections of skin phototypes III and IV: interest of the Beautyqol instrument. Clin Exp Cosm Derm J 2017;1:1-4.

29 Chen S-C, Huang B-S, Lin C-Y, et al. Psychosocial effects of a skin camouflage program in female survivors with head and neck cancer: a randomized controlled trial. Psychooncology 2017;26:1376-83.

30 Matsuoka Y, Yoneda K, Sadahira C, et al. Effects of skin care and makeup under instructions from dermatologists on the quality of life of female patients with acne vulgaris. J Dermatol 2006;33:745-52.

31 Nicoletti G, Sasso A, Malovini A, et al. The role of rehabilitative camouflage after cervicofacial reconstructive surgery: a preliminary study. Clin Cosmet Investig Dermatol 2014;7:43-9.

32 Richard A, Harbeck N, Wuerstlein R, et al. Recover your SMILE: effects of a beauty care intervention on depressive symptoms, quality of life, and self-esteem in patients with early breast cancer. Psychooncology 2019;28:401-7.

33 Finlay AY, Khan GK. Dermatology Life Quality Index (DLQI)--a simple practical measure for routine clinical use. Clin Exp Dermatol 1994;19:210-6.

34 APA Presidential Task Force on Evidence-Based Practice. EvidenceBased practice in psychology. Am Psychol 2006;61:271-85.

35 Kwakkenbos L, Jewett LR, Baron M, et al. The scleroderma patientcentered intervention network (spin) cohort: protocol for a cohort multiple randomised controlled trial (cmRCT) design to support trials of psychosocial and rehabilitation interventions in a rare disease context. BMJ Open 2013;3:e003563.

36 Donker T, Blankers M, Hedman E, et al. Economic evaluations of internet interventions for mental health: a systematic review. Psychol Med 2015;45:3357-76.

37 Andersson G, Cuijpers P, Carlbring P, et al. Guided Internet-based vs. face-to-face cognitive behavior therapy for psychiatric and somatic disorders: a systematic review and meta-analysis. World Psychiatry 2014;13:288-95.

38 Heber E, Ebert DD, Lehr D, et al. The benefit of Web- and computerbased interventions for stress: a systematic review and metaanalysis. J Med Internet Res 2017;19:e32.

39 Stiggelbout AM, Van der Weijden T, De Wit MPT, et al. Shared decision making: really putting patients at the centre of healthcare. BMJ 2012;344:e256. 